\title{
Agile Software Development with Open Source Software in a Hospital Environment - Case Study of an eCRF-System for Orthopaedical Studies
}

\author{
Tünay Özcan ${ }^{1,2}$, Semra Kocak ${ }^{1}$, and Philipp Brune ${ }^{2}$ \\ 1 Orthopädische Studienzentrale des Universitätsklinikums \\ Oberer Eselsberg 45, D-89081 Ulm \\ 2 Hochschule Neu-Ulm \\ Wileystraße 1, D-89231 Neu-Ulm \\ tuenay_oezcan@yahoo.com , \\ semra.kocak@rku.de, \\ philipp.brune@hs-neu-ulm.de
}

\begin{abstract}
In recent years, agile development of web-based applications as well as open-source software (OSS) have been subject to research and practical application in many domains. For the healthcare sector, the use of OSS has been studied in the literature with contradicting findings. Regarding OSS in clinical applications, mainly case studies from hospital-wide IS have been reported. Agile methods have been examined merely in the context of healthcare software product development. However, the development of web-based applications in clinical departments using agile methods and OSS has not been studied so far. Thus, in this paper the feasibility of such an approach is examined for an electronic case report form (eCRF) application for orthopaedical studies. It is demonstrated how OSS-based web engineering projects may be successfully accomplished in highly specialized environments like clinical departments by properly taking into account their specific requirements.
\end{abstract}

Keywords: Web Application Engineering, Open Source Software, Agile Software Development, Healthcare IS, Clinical Studies.

\section{Introduction}

The healthcare sector in general and especially clinical centers in the western world are currently facing increasing economical pressure due to medical progress and demographic change. The lack of financial and personal resources forces healthcare providers to strongly optimize their processes in order to cope with these changes. However, all optimizations need to take into account the high quality standards required for medical treatment [5]. Therefore, information systems (IS) play an important role, i.e. electronic patient record (ePR) systems $[5]$. 
The German federal association for healthcare IT (Bundesverband Gesundheits-IT) in 2011/2012 conducted a survey among stakeholders in hospitals to identify IS trends and measure user satisfaction. On the question "which intranet/internet application would become increasingly important within the next 5 years" for them, 82 of 100 interviewed physicians answered that state-of-theart medical knowledge will become more and more important. To collect and distribute this knowledge by scientific publications, clinical studies are required.

Clinical studies require to collect and store the underlying empirical data in a structured and persistent form. Therefore, information technology plays an important role. eCRF applications allow to perform patient surveys in electronic form and support the related data management and administrative tasks. During the development of an eCRF application it is especially important to focus on the user requirements, in particular those of the patients and physicians, to ensure an efficient process support and a high user acceptance of the resulting software. Thus, in the present case a web-based eCRF application for orthopaedical clinical studies was developed individually by and for the center for orthopaedical studies (Orthopädische Studienzentrale) of the Ulm university hospital. Due to the close interaction with the relevant stakeholders (in particular physicians) and the unclear detail requirements at project start, an agile method based on Scrum was chosen for the project [/24/32].

To ensure long-time support and operation, an application also needs to adhere to the standards and requirements of the IT organization. Therefore, beside the selection of an appropriate development method, the application architecture and the proper choice of its technical components are crucial for the application's success [27]. Here, open source software (OSS) provides a possible alternative to commercially available solutions. Besides the savings of the licence fees, OSS usually offers good transparency, extendability and the support of open standards [13. In the literature, agile software development of web-based applications not only for but in a clinical department in combination with OSS has not been studied so far [3. Thus, this paper analyses the feasibility of such an approach by examining the proof-of-concept implementation of the real eCRF system OrthoClinical. In addition, the usability of OSS components as a basis for designing the software architecture of web-based applications is analysed in this context.

The rest of this paper is organized as follows: In section 2 the related work is reviewed and section 3 describes the project context of the proof-of-concept implementation of OrthoClinical. Section 4 illustrates the selection and customization of the agile concepts used, and in section 5 the application architecture including the selected OSS components is presented. In section 6 the results of the evaluation of the proof-of-concept prototype are discussed in detail. We conclude with a summary of our findings.

\section{Related Work}

Since the introduction of Extreme Programming (XP) [6] and the declaration of the agile manifesto [7, agile methods have been intensively discussed in research 
and practice [12. Popular agile methods today include the Crystal family [1] and in particular Scrum 32 . All these methods more or less form a set of best practices, which have to be customized according to the requirements of each project [2].

Reasons for the success of Scrum and other agile methods have been studied by various authors [2910 25]. In [12, 36 studies regarding agile methods are examined, mainly related to XP, which is found to be inadequate for larger organizations. Hence, agile methods show to be most effective with smaller teams and social factors being the most important influence on project success 12 .

One example reported for the use of Scrum in the healthcare sector is a pilot project related to imaging diagnostic systems conducted by GE Healthcare corporation 3 . In the context of critical systems development in general, agile methods and OSS have been also examined, but not explicitly with respect to the healthcare sector [15].

In recent years, the use of OSS in the healthcare sector has been studied by different publications. Most of the authors focus on the general economical, organizational and technical advantages and disadvantages of OSS, with heterogeneous findings [1127/3 28]. Especially in Europe, a limited acceptance of OSS in the healthcare sector is observed [13]. The majority of the examined clinical OSS projects were not completed due to inactivity already in early stages. However, these projects mainly were related to the development of hospital information systems (HIS) and imaging diagnostics [16.

Various authors report case studies on individual OSS projects, i.e. the redesign of the Irish Beaumont hospital's IT infrstructure [14, the Decentralized Hospital Computer Program (DHCP) system [8] and its successor VistA [920, clinical decision support systems [3431], the Mediboard system in Mali [4, a private cloud platform for healthcare data in India [17, or the MyHealthService project [35]. In addition, there exist some projects related to imaging diagnostics based on OSS, i.e. an approach for image processing based on the Digital Imaging and Communications in Medicine (DICOM) standard 18. Another example is PrivacyGuard, a tool supporting the anonymization of clinical image data [30.

However, the literature on applications in the clinical research domain is sparse. With respect to eCRF systems, only the project PHPSurveyor was studied. Originally developed for a special disease pattern, it may be also used in other contexts due to its modular design [23]. Regarding the orthopaedic domain, Shah et al. have analyzed the existing Electronic Data Capture (EDC) systems available commercially or as OSS 33. They state that the majority of the clinical departments still is using inefficient spreadsheet applications to support clinical studies, which is considered inflexible, insecure and inefficient with respect to process support. Also the data is stored that way in a non-standard and poorly structured form, thus prohibiting data exchange from the beginning. In addition, it is observed that some physicians are still not willing to share their data with others, despite the medical benefits of such data collections. Reasons are considered to be a lack of physicians' resources and missing IT support in form of adequate EDC systems. Therefore, the authors recommend the 
data collection on the level of individual institutions by means of databases in combination with intuitive and user-friendly front-end systems 33 .

Thus, it can be concluded that the use of agile methods in combination with OSS in the healthcare sector has rarely been studied in the literature. In particular, projects implementing individual web-based applications in the domain of clinical research and their integration with the hospital-wide IT organization have not been reported so far at all. In addition, only very few case studies from the German or European regions even about partial aspects of the mentioned topics exist. Thus, in the present work it is examined by studying the implementation of the OrthoClinical eCRF system at the Ulm university hospital, how:

- agile methods may be adapted to the clinical domain to enable rapid development of efficient applications,

- a web-based orthopaedical eCRF system with integrated study management module may be developed based on OSS, and which OSS components may be selected for its architecture in order to fulfill the requirements regarding cost efficiency, interoperability, rapid development and standard conformance.

\section{Project Context}

The RKU - Universitäts- und Rehabilitationskliniken Ulm (University and Rehabilitation Clinics Ulm) are maximum care clinics focussing on orthopaedics and neurology. Founded in 1969 by the Ulm university hospital, today the clinics offer acute care and subsequent medical and professional rehabilitation of orthopaedic and neurological patients. In addition, the clinics support teaching and research at the university hospital. Therefore, some of the physicians working at RKU are directly employed by the university hospital. In total, RKU has a staff of 500, including 60 employees in medical service and 176 in nursing.

The orthopaedical department founded the center for orthopaedical studies in 2011 to support the research activities of the physicians. It supports the data collection, extraction and analysis for clinical studies among ambulant patients. Located at RKU, the center organizationally belongs directly to the university hospital. So far, the data for clinical studies was collected by means of paperbased questionnaires handed out to the ambulant patients. Then, the filled-out questionnaires were manually recorded in a spreadsheet application and subsequently analyzed with standard statistics software tools. To enable more efficient research, the implementation of an adequate software solution for electronic data collection and processing was necessary. In a first step, this system should be only used by the center for orthopaedical studies and primarily help to reduce the workload of the physicians with respect to the extension of the clinical study database.

This paper describes the development of a first prototype of this application, called OrthoClinical. For an easy access by different types of client devices (mobile and stationary), OrthoClinical was designed as web-based application. 
The prototype was required to support at least the design and administration of electronic questionnaires and the electronic execution of patient surveys (i.e. in the patients' waiting area by means of a tablet computer). Later, it should be extended to a full-featured eCRF system supporting retrospective as well as prospective studies.

\section{Agile Methodology}

The selection of an adequate project methodology was performed by means of the decision matrix proposed by Cockburn [1. The priorities of the project were considered to be productivity and tolerance, since first results should be available at an early stage, which the team atmosphere should enable. Thus, the productivity and tolerance grid was chosen as basis for the decision (see Fig. 1).

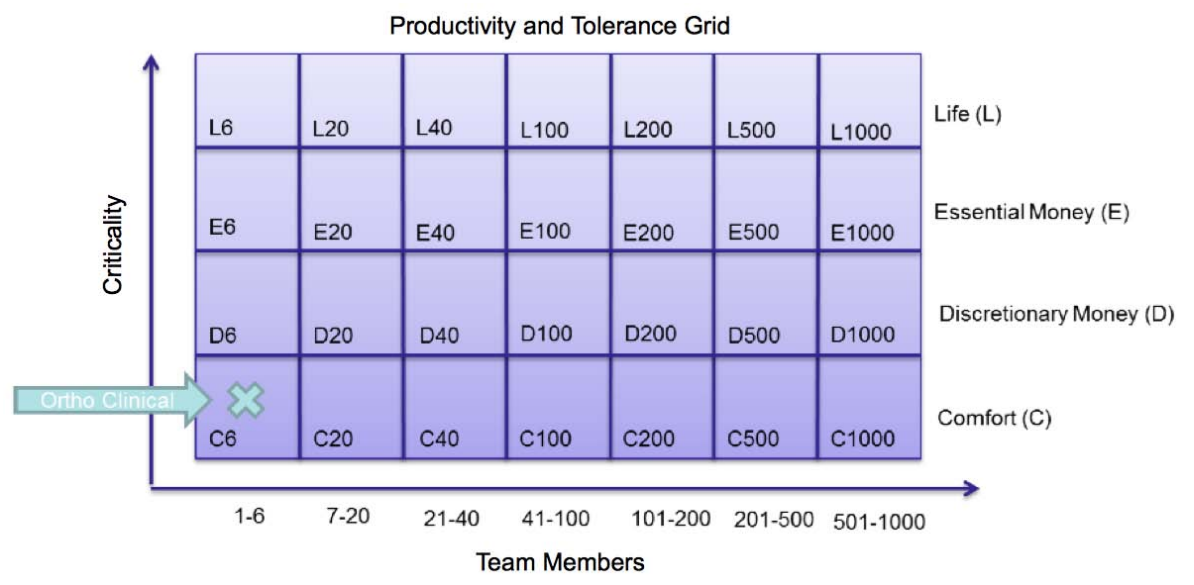

Fig. 1. Decision matrix for project methodology selection according to Cockburn [1]

The project's criticality was classified as "Comfort", since the system only reduces the workload of the users. The development team consisted of 3 persons. Thus, according to Cockburn it is a C6 project requiring cost-effective development, since no direct financial benefits are expected, frequent delivery of working software and a high demand for communication between the team and other stakeholders.

To meet this requirements a combination of the agile methods Crystal Clear and Scrum was chosen. Usually Scrum is considered to requires an experienced team with good technical skills in the development environment used. Additionally, a close interaction with the customer representatives is needed, i.e. for testing the delivered software after each sprint [10]. However, these requirements were not completely met by the project. Neither experienced developers were available, nor the customer representatives (physicians) were able to perform 
a regular quality assurance after each sprint due to their tight schedules and shifted working times.

According to Crystal Clear, the "Exploratory 360" strategy was used at project start [2]. Thereby, the items business value, requirements, technology plan, team make up and working conventions were analyzed and defined. The category domain model was integrated into the working conventions and not considered separately.

First, together with the stakeholders the business value definition was worked out [2]. Therefore, interviews with the assistant medical director, physicians, medical documentation officers and MD students were done to estimate the system's expected value for the orthopaedical department. It became obvious that the system should not only improve the efficiency of the processes but also increase the transparency about the research activities at RKU. For a cost-efficient implementation it was decided to develop the application based in OSS. To support different client devices, the implementation as a web-based application was chosen.

The high-level use cases related to administration, data capture and scheduling were identified to be the most important requirements, therefore these functional domains were implemented as core modules of OrthoClinic. Fig. 2 illustrates this modular structure. The high-level use cases were then refined and worked out in form of user stories. In discussions with the stakeholders it become clear that the modules administration and data capture were of highest priority, thus it was decided to implement them first.

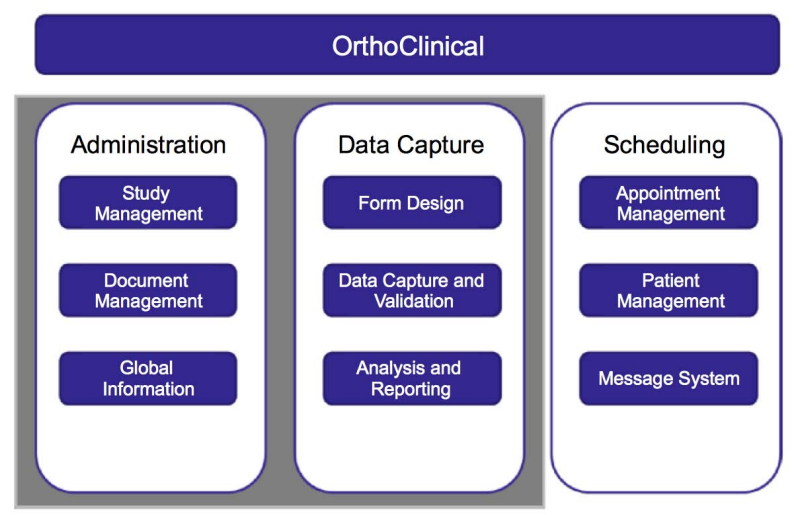

Fig. 2. Functional modules of OrthoClinical

Crystal Clear defines the project roles executive sponsor, expert user, lead designer, designer-programmer and coordinator, the latter being responsible for project management. In smaller teams it is necessary for the members to take multiple of these roles [2]. Therefore, during the team make-up of the OrthoClinical project the roles sponsor, coordinator, lead designer, designer-programmer 


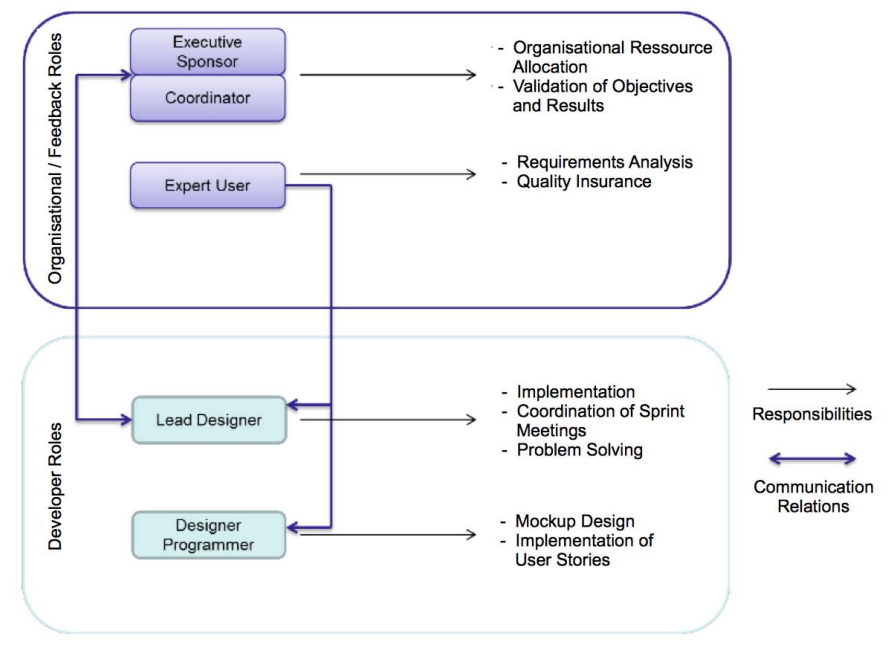

Fig. 3. Project roles according to Crystal Clear with the respective communication relations and responsibilities

and expert user were assigned. The role of the expert user was taken first by a medical specialist, and second by a staff member of the center for orthopaedical studies. Fig. 3 illustrates the assigned project roles with their respective communication relations and tasks within the project.

Working conventions help to organize the daily work of the team. Therefore, Crystal Clear defines seven categories, namely frequent delivery, reflective improvement, osmotic communication, personal safety, focus, easy access to expert user und technical environment. Osmotic communication and easy access to expert user proved to be especially challenging in the present project context.

Osmotic communication should allow the developers to take part directly or indirectly in every development-related communication process. However, since in the present scenario not all developers were able to work at the same time at the project, an indirect form of osmotic communication was established by means of an information board (see Fig. 4). Here, i.e. coding standards or informations about development items were put on visible to all team members. Also problems or impediments could be noted and discussed on the board by all developers. Thereby it was possible to discuss ideas or solutions without the need for all team members to be physically present all the time.

The easy access to an expert user also proved to be a challenge to the project. Crystal Clear requires the expert user to be continuously involved in the development process as close as possible to ensure that deviations between the current implementation and the requirements are identified quickly. However, in the present case this principle needed to be adapted. At project start a physician was denoted as the expert user. However, due to the tight schedules and shifted working times of the physicians this proved not to be feasible. Instead, the role of the expert user was additionally taken by a medical documentation officer, who 


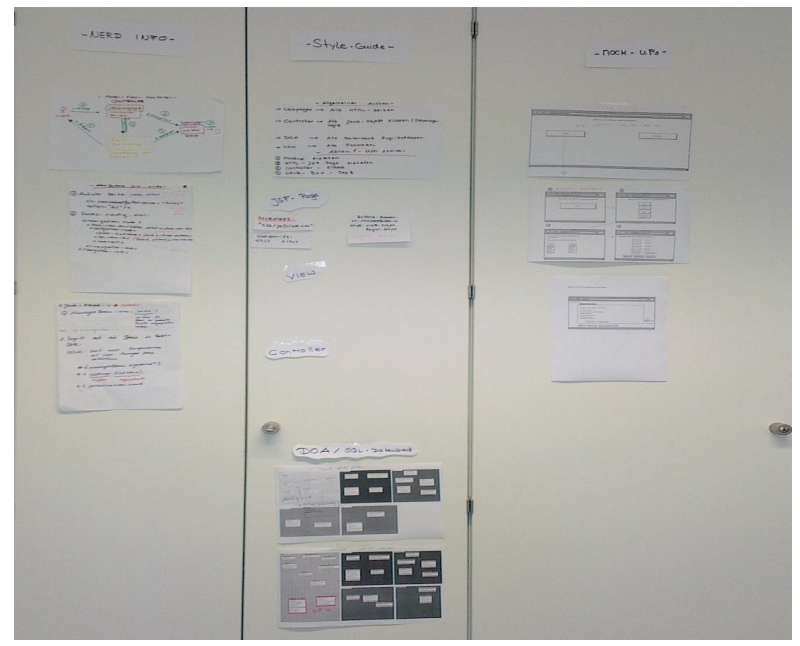

Fig. 4. Snapshot of the information board used for communication between the team members

was also involved in the development himself. Thus, no continuous independent quality assurance could be guaranteed.

The actual development work was organized using Scrum. Before the start of the first iteration (sprint) already all major requirements needed to be identified. First, since the physician serving as the expert user was not available during the rest of the project, and second because it is required for software projects at RKU to deliver a detailed IT security and privacy concept at project start.

The functional requirements were specified as user stories collected in interviews with selected physicians and medical documentation officers. To further involve the other physicians and obtain their feedback, the collected user stories were distributed to all of them in written form. In total, 22 user stories were identified and put into the product backlog. According to Scrum, the user stories in the product backlog should be prioritized by the product owner. The role of the product owner in the project was taken by different persons, first by the project sponsor, then for pragmatic reasons by the expert user and the lead programmer.

So far, three sprints were performed, each lasting for one week. Sprint meetings were scheduled on demand and after each sprint. Due to time limitations not all user stories could be implemented as planned during these sprints. After the first sprint the prioritization and number of relevant user stories was adjusted. Scrum requires to involve the users in the testing and approval of the implemented user stories after each sprint. This proved not to be feasible in the clinical context. In the future, the regular early morning status meetings of the physicians might be used for testing individual functionalities and for general information about the project progress. This would not only allow a better individual feedback by the physicians, but also provide an environment for broader discussions with the users. 


\section{Application Architecture and OSS Components}

OrthoClinical was realized as a web-based application with a standard three-tier architecture, composed of the database, business logic and presentation layers. Therefore, in general different OSS platforms may be considered, in particular the frequently used PHP and Java Enterprise Edition (EE) environments [22], since for the latter also different open source implementations exist. Criteria for the selection of the used components were license fees, initial skill adaptation effort, interoperability, a high productivity within short development cycles and support for open standards. From these, the initial skill adaptation effort strongly depends on the experience and knowledge of the team members. Thus, no general statement for other projects is possible here.

Both Java EE and PHP fulfill the selection criteria license fees, interoperability and high productivity. PHPSurveyor, the only OSS-based eCRF web application documented in the literature so far, was developed using PHP 23 . However, for OrthoClinical some significant disadvantages of PHP were identified: First, the initial skill adaptation effort would have been higher for PHP, since the team members already had Java EE but little PHP development experience. Second, PHP in contrast to Java EE does not provide a dedicated support for business logic implementation by a built-in component architecture. Due to the lack of type safety, for PHP also a higher testing effort was expected. In contrast, Java EE with its Enterprise Java Beans (EJB) component architecture provides a solid foundation for implementing business logic. With Eclipse and Netbeans IDE 21] also powerful development environments exist for Java EE.

Thus, Java EE and Java ServerFaces (JSF) 2.0 were selected as the development platform [22. The standard JSF framework was extended for OrthoClinical by the Primefaces library. Using JSF and the Primefaces UI library [26] enabled the developers to create a professional web user interface by combining visual components. The communities of both frameworks are very active and questions could be discussed with members in various forums. JSF is well documented. In contrast, Primefaces indeed offers many realistic examples, but lacks a comprehensive open online documentation.

Enterprise Java Beans (EJB) like JSF is a standardized technology. This is an advantage regarding extendability and maintainability. The data access layer was implemented using Java Persistence API (JPA) and EclipseLink for objectrelational mapping (ORM) 22. The database layer was realized by the popular database management system (DBMS) MySQL 5.2.

NetBeans 7.2 with its integrated GlassFish application server was used as development environment. It provides i.e. a powerful scaffolding functionality for automatically generating business logic classes (EJB) and JSF pages from a relational database model 21]. For the creation of this database model and the administration of the underlying DBMS the MySQL Workbench software was used. The scaffolding functionality of NetBeans IDE proved to be extremely useful. A disadvantage was the instability of the integrated GlassFish server. As a workaround, the server subsequently was installed and administrated separately from NetBeans. 


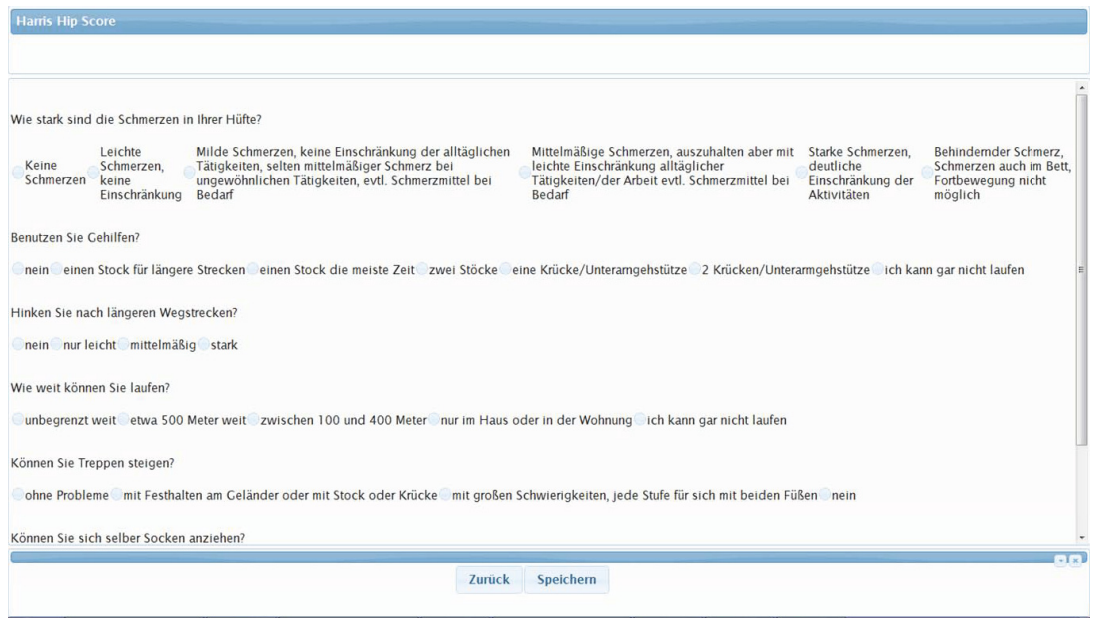

Fig. 5. Screenshot of a questionnaire form (Harris Hip Score, in German language) in OrthoClinical

Fig. 5 shows a screenshot of a patient questionnaire form (Harris Hip Score) in the resulting OrthoClinical web application developed this way.

\section{Prototype Evaluation}

In retrospect, the developers consider the selected OSS implementation of the Java EE technology stack and development tools as mature and effective means to build user-friendly and reliable web-based applications. In particular the scaffolding functionality of Netbeans IDE for generating skeleton JSF pages and presentation and business layer Java Beans in combination with the Primefaces UI library strongly supported the rapid development of working increments of the software during the sprints.

To validate the practical usability of OrthoClinical for patient surveys, an empirical usability test was performed. Therefore, 14 randomly selected patients in the orthopaedical department were asked to fill out the questionnaire for the so-called Harris Hip Score in OrthoClinical (see Fig. 5) using a tablet PC. All participants were asked to perform a representative and identical task in OrthoClinical, namely to "fill out the Harris Hip Score questionnaire for the hip body region". They did not receive any special training on how to use OrthoClinical before. The objective was to measure the usability criteria effectiveness, efficiency and user satisfaction. Effectiveness indicates if the users are able to perform a certain task successfully, efficiency measures the effort necessary for it. User satisfaction indicates the subjective perception of the usability by the test persons. The last criteria is only meaningful with respect to the average user satisfaction of the respective peer group [19]. During the tests the patients were 
observed and the durations to complete the test task were measured. A successful completion of the test task required the participants to perform the following steps: 1. selection of the patient region in OrthoClinical, 2. fill out the questionnaire, 3. save the results. After the test, the participants were interviewed to measure the usability criteria. The results are shown table 1 .

Table 1. Results of the usability test of OrthoClinical from the patients' perspective

\begin{tabular}{|l|l|l|l|}
\hline Age & Effectiveness & Efficiency & User Satisfaction \\
\hline $25-45$ & Yes, without support & $3.5 \mathrm{~min}$. & High \\
\hline $45-65$ & Yes, with support & $4.5 \mathrm{~min}$. & Medium \\
\hline $65-75$ & No, despite support & $<8 \mathrm{~min}$. & Low \\
\hline
\end{tabular}

The results show that older patients in general had problems using electronic client devices, regardless of the user-friendly web-based frontend. Two patients even refused to take part in the test because they never had used such devices in their everyday life. Some participants also criticised the current way how questionnaires are displayed in OrthoClinical (all question on one web page). They suggested that there should be a separate page for answering each question. On the positive side, the overall GUI layout of the web pages was considered well structured.

Participants between 25 and 35 years were able to perform the task without support in an average of 3.5 minutes. The 45 to 55 year old patients partially needed help, i.e. regarding the scrolling of the questionnaire page. The user satisfaction in this user group was medium. The test persons older then 65 years had significant problems using OrthoClinical. Despite personal assistance they were not able to successfully complete the task. As a reason for this an overall lack of computer skills was mentioned.

\section{Conclusion}

In conclusion, the project demonstrated that it is in general possible to use agile methods for web-based application development in a clinical environment. However, these methods need to be customized and extended with respect to the identified specific requirements. Due to the required quality insurance measures at project start it was not possible to use agility during all phases of the development process. To get an approval for the project by the IT administration it was i.e. necessary to create an technical specification and IT security document in advance, which already defined key functionalities, the software architecture, and the data model. Nevertheless it was possible for the rest of the project to design and use a tailored agile methodology combining elements of Scrum and Crystal Clear. 
The OSS components used developing the OrthoClinical web application proved to provide an efficient alternative to commercially available products. Originally, the main reason for the clinics administration to use OSS was the savings of license fees. However, during the project also further advantages like extendability and vendor independence became apparent.

However, further research is necessary to evaluate the presented approach more extensively for a longer duration, and also in various similar project contexts.

\section{References}

1. Cockburn, A.: Selecting a projects methodology. IEEE Software 4, 64-71 (2000)

2. Cockburn, A.: Crystal Clear - A human powered methodology for small teams. Addison-Wesley (2005)

3. Deitsch, A., Hughes, R.: Ge healthcare goes agile. Information Week, 59-63 (2010)

4. Bagayoko, C.: Selection of secure single sign-on solutions for heterogeneous computing environments. BMC Medical Informatics and Decision Making 10(22), 1-13 (2010)

5. Bärwolff, H., Hüsken, V., Victor, F.: IT-Systeme in der Medizin IT- Entscheidungshilfe für den Medizinbereich - Konzepte, Standards und optimierte Prozesse. Vieweg+Teubner Verlag, Wiesbaden (2006)

6. Beck, K.: Extreme Programming eXplained - Embrace Change. Addison-Wesley (2000)

7. Beck, K., et al.: Principles behind the agile manifesto (2001), http://agilemanifesto.org/principles.html

8. Brown, S., et al.: Vista u.s. department of veteran affairs national scale his. International Journal of Medical Informatics 69, 135-156 (2003)

9. Byrne, C., et al.: The value from investments in health information technology at the u.s. department of veteran affairs. Health Affairs 29(4), 1-13 (2010)

10. Cesar, A., et al.: An empirical study on the relationship between use of agile practices and the sucess of scrum projects. In: Proc. of the 2010 ACM-IEE International Symposium on Empirical Software Engineering and Measurment (ESEM 2010), vol. $10(2010)$

11. Dinevski, D., Inchingolo, P., Krajnc, I., Kokol, P.: Open source in health care and open three example. In: IEEE International Symposium on Combuter Based Medicine (2007)

12. Dyba, T., Dingsor, T.: Empirical studies of agile software development: A systematic review. Information and Software Technology 50, 833-859 (2010)

13. Murray, P., et al.: Open source and healthcare in europe time to put leading edge ideas into practice. Medical Informatics in a United and Healthy Europe (2009)

14. Fitzgerald, B., Kenny, T.: Open source software in the trenches: Lessons from a large-scale oss implementation. In: 24th International Conference on Information Systems (ICIS), pp. 316-326 (2003)

15. Gary, K.: Agile methods for open source safety critical software. Software Practice and Experience, 943-962 (2011)

16. Hogarth, M., Turner, S.: A study of clinilally related open source software projects. In: AMIA Annu. Symp. Proc., pp. 330-334 (2005) 
17. Lakshimi, M., Malar, J.D.: An open source private cloud solution for rural healthcare. In: International Conference on Signal Processing Communication Comouting and Networking (2011)

18. Marcheschi, P., Mazzarisi, A., Benassi, A., Ferdeghini, E.: An open source based radiological information system to support a clinical cardiology depearment. In: Computer in Cardiology, pp. 363-366 (2003)

19. Hartmann, M.: Usability Untersuchungen eines Internetauftrittes nach DIN EN ISO 9242 Am Praxisbeispiel der Firma MAFI Transport Systeme GmbH. Diplomica Verlag GmbH (2008)

20. Molin, J.D.: Open innovation; transforming health systems trough open and evidence based healthcare ict innovation. Communication \& Strategy 3, 17-35 (2011)

21. Netbeans: The netbeans e-commerce tutorial (2012), http://netbeans.org/kb/docs/javaee/ecommerce/entity-session.html

22. Oracle: The java persictence api a simpler programming model for entity persistence (2012), http://www . oracle.com/technetwork/articles/javaee/jpa-137156.html

23. Orr, S., Straus, S., Holyrod-Leduc, J.: Development and example of a web based open source clinical tool. In: AMIA 2005 Symposium, pp. 330-334 (2005)

24. Pichler, R.: Scrum agiles Projektmanagement erfolgreich einsetzen. Dpunkt Verlag (2008)

25. Pries-Heje, L., Pries-Heje, J.: Agile and distributed project management: A case study revealing why scrum is useful. In: ECIS 2011 Proceedings, vol. 217 (2011)

26. Primefaces: Ultimate jsf component suite (2012), http://www.primefaces.org

27. Ralston, B.: Open source expected to improve innovation. Health Management Technology 30(8), 12-13 (2011)

28. Reynolds, C., Wyatt, J.: Open source, open standards and health care information systems. J. Med. 13(1) (2011)

29. Rising, L., Janoff, N.: The scrum software development process for small teams. IEEE Softw. 17(4), 26-32 (2000)

30. Rodrigez, D.G., Carpenter, T., van Hemert, J., Wardlaw, J.: An open source toolkit for medical imaging de-identification. Eur. Radiol., 1896-1903 (2010)

31. Sackett, D.: Implementing an integrative mulitagent clinical decision support system with open source software. J. Med. Sys. 36(1), 123-137 (2012)

32. Schwaber, K.: Scrum alliance - scrum guide (2009), http://www.scrum.org/Scrum-Guides

33. Shah, J.: Electronic data captuare for registries and clinical trails in orthopaedic surgery: Open source versus comercial systems. Clin. Orthop. Relat. Res. 468(10), 2664-2671 (2010)

34. Shirabad, S.: Implementing an intergrative multi-agent clinical decision support system with open source software. J. Med. Syst., 123-127 (2010)

35. Vognild, L., Fernandez, L., Burkow, T.: The myhealth service approach for chronic disease management based on free open source software und low cost components. In: 31st Annual International Conference of the IEEE EMBS, pp. 1234-1237 (2009) 